\title{
Hepatocellular carcinoma recurrence after directly acting antivirals for chronic hepatitis C: a 2-year follow-up study
}

\author{
Ahmed Kamal', Amr Aly Abd Elmoety', Yousri Abdelmeguid Rostom², Mohamed Said Shater' ${ }^{1}$ Sameh Aldesoky Lashen \\ 'Hepatology Unit, Internal Medicine Department, Alexandria University, Egypt \\ ${ }^{2}$ Clinical Oncology and Nuclear Medicine Department, Alexandria University, Egypt
}

\begin{abstract}
Aim of the study: Data regarding hepatocellular carcinoma ( $\mathrm{HCC}$ ) recurrence after directly acting antivirals for hepatitis $\mathrm{C}$ are contradictory. Our aim was to study the $\mathrm{HCC}$ recurrence in patients who received directly acting antivirals after tumor ablation.

Material and methods: This retrospective study included all Child-Pugh $A$ and $B$ patients with hepatitis $C$ related $<5 \mathrm{~cm}$ single or up to $3 \mathrm{HCC}$ without any vascular or extrahepatic involvement whose lesions were managed using microwave or radiofrequency ablation at the Internal Medicine Department of Alexandria Faculty of Medicine, in the period from 1 January 2016 to 31 December 2016, and then received directly acting antivirals.

Results: Data from 52 patients were analyzed. Throughout the 2 years from ablation, $42.3 \%$ of patients experienced tumor recurrence (22 out of 52 patients). In addition, two subjects died and 4 subjects were lost to follow-up before any tumor recurrence.

Conclusions: Although our study included both modified Child-Pugh A and B patients and included lesions up to $5 \mathrm{~cm}$ treated using thermal ablation, the 2-year $\mathrm{HCC}$ recurrence rate was similar to that previously reported after surgical resection or radiofrequency ablation of lesions up to $3 \mathrm{~cm}$ in Child-Pugh A patients before development of directly acting antivirals.
\end{abstract}

Key words: hepatocellular carcinoma, chronic hepatitis C, antiviral agents, liver cirrhosis, neoplasm recurrence.

Address for correspondence:

Dr. Ahmed Kamal, Hepatology Unit, Internal Medicine Department, Faculty of Medicine, Alexandria University, Egypt, e-mail: ahmed.kamal@alexmed.edu.eg

\section{Introduction}

Hepatocellular carcinoma (HCC) is the fifth most frequent cancer globally [1] and the most frequent cancer in Egypt, comprising 23.8\% of all malignancy cases, because of high hepatitis $\mathrm{C}$ virus (HCV) prevalence [2].

Antivirals for hepatitis B virus (HBV) have a known contribution to decreasing HCC recurrence [3]. Unfortunately, the condition is different regarding HCV. Many studies have been performed to study the effect of HCV clearance on HCC recurrence in the era of pegylated interferon [4]. The long-term studies found a reduction in the incidence of HCC over time as a result of switch-off of the necro-inflammatory process and inflammatory cyto- kines, but without complete elimination of the risk, which indicates that long-term surveillance is necessary [5].

Data regarding HCC recurrence after directly acting antiviral (DAA) treatment are contradictory. Surprisingly, some studies have reported that DAAs are associated with higher HCC recurrence [6-10]. However, other studies showed no rise in HCC recurrence [11-13]. Our aim was to study the 2-year recurrence rate of HCC in HCV patients who were treated using radiofrequency ablation (RFA) or microwave ablation (MWA) at our unit from 1 January 2016 to 31 December 2016 and then received DAAs. Both techniques have comparable results according to a paper previously published by our group [14]. 


\section{Material and methods}

\section{Materials}

This retrospective study included all Child-Pugh A and $B$ subjects with hepatitis $C$ related $<5 \mathrm{~cm}$ single or up to $3 \mathrm{HCC}$ without any vascular or extrahepatic involvement whose lesions were managed using MWA or RFA at the Internal Medicine Department of Alexandria Faculty of Medicine in the period from 1 January 2016 to 31 December 2016 and then received DAAs. Only those with LR5 lesions according to the Liver Imaging Reporting and Data System (LI-RADS) classification were considered [15]. The number of patients meeting the inclusion criteria was 189 . Those without sufficient clinical data or who did not start HCV treatment within the first year after tumor ablation, those with positive hepatitis B surface antigen ( $\mathrm{HBsAg}$ ), any other known cause of chronic liver disease, ongoing alcohol consumption and human immune deficiency virus (HIV) infection were excluded. Also patients who had received DAAs before occurrence or treatment of HCC and those with previous treatment for HCC were excluded. Then data from the remaining 52 patients were analyzed (Fig. 1). The study was conducted in accordance with the Declaration of Helsinki and was ap- proved by the Alexandria Faculty of Medicine human research ethical committee.

\section{Methods}

Data were collected regarding history, clinical findings, complete blood count (CBC), serum aspartate and alanine aminotransferases (AST and ALT), serum bilirubin, serum albumin, prothrombin activity, international normalized ratio (INR), $\alpha$-fetoprotein (AFP), $\mathrm{HCV}$ antibodies, $\mathrm{HBsAg}$ and hepatitis $\mathrm{B}$ core antibody ( $\mathrm{HBcAb})$ using ELISA, HCV RNA levels in serum using real-time PCR assay and HCV genotyping. Liver disease severity was assessed based on the modified Child-Pugh score and the Model for End-Stage Liver Disease (MELD) score within 2 weeks before the ablation procedure. Radiological assessment relied on triphasic computed tomography (CT) liver scan and/or dynamic magnetic resonance imaging (MRI) of the liver carried out within 4 weeks prior to ablation.

A RITA StarBurst XL needle and Angiodynamics RITA model $1500 \times$ (USA) generator were used for RFA while a 14 gauge $200 \mathrm{~mm}$ AMICA probe $\mathrm{MW}$ and a $2.45 \mathrm{GHz}$ AMICA GEN AGN-H-1.2 (Italy) generator were used for MWA with adherence to the manufacturer's instructions.

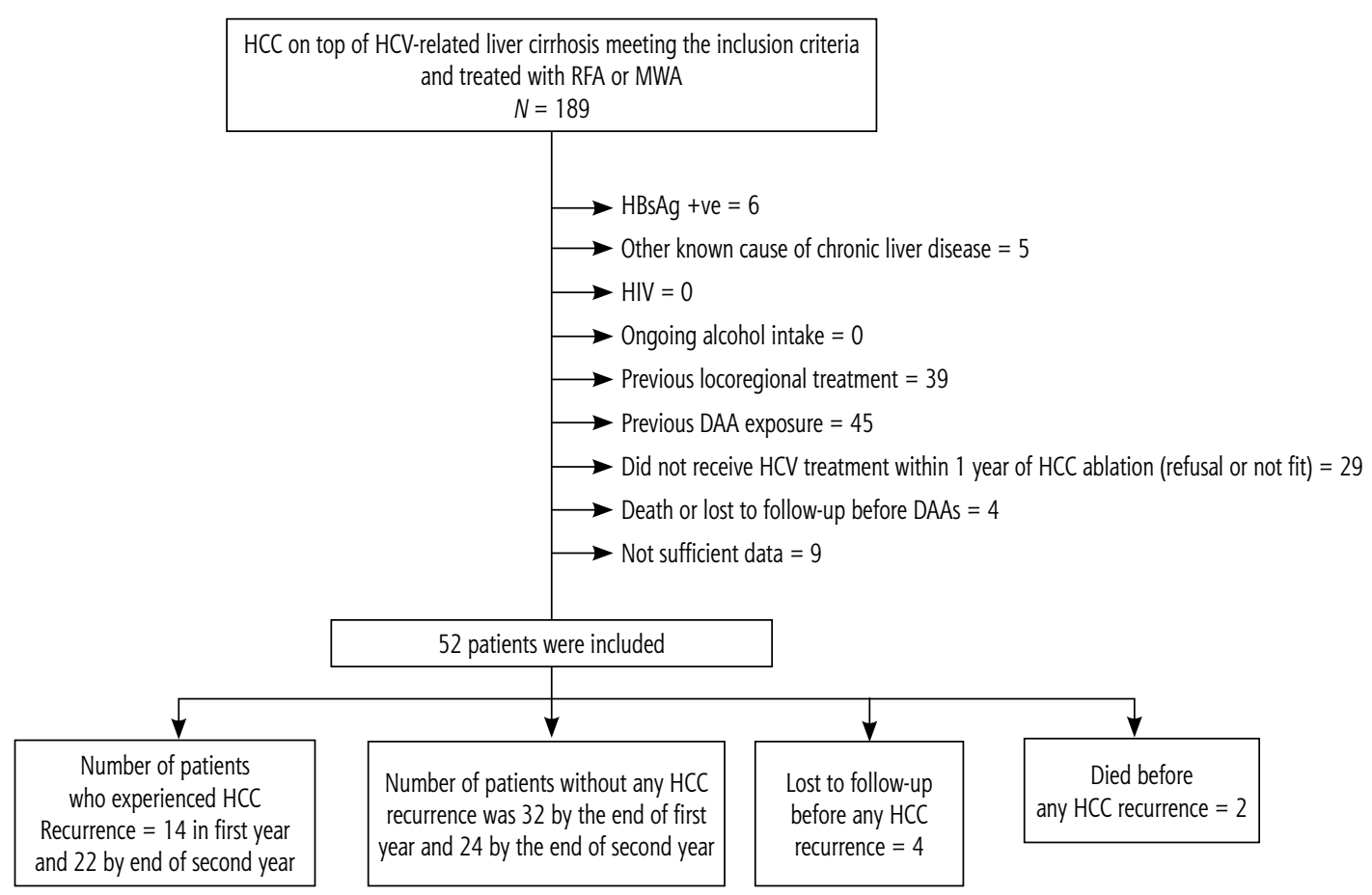

HCC - hepatocellular carcinoma, HCV - hepatitis C virus, RFA - radiofrequency ablation, MWA - microwave ablation, HBsAg - hepatitis B surface antigen, HIV - human immune deficiency virus, DAA - directly acting antiviral

Fig. 1. Flow chart of the study 
Local response was evaluated by a triphasic CT liver scan 4 weeks after the ablation session. If there was any residual activity, retreatment using RFA or MWA was performed and another triphasic CT scan was performed after a further 4 weeks. No one required more than 2 sessions to achieve complete ablation.

The regimen of DAAs was chosen for each patient according to European Association for the Study of the Liver (EASL) recommendations 2015 [16].

Triphasic CT scans were performed every 3 months from the procedure to reveal any tumor recurrence. Response was assessed according to the modified Response Evaluation Criteria In Solid Tumors (mRECIST) criteria [17]. HCC recurrence was defined as either local recurrence or development of new HCC lesion(s).

\section{Statistical analysis}

IBM SPSS software package version 20.0 was used. Numbers and percent were used to describe qualitative data. Normality of distribution was assessed using the Kolmogorov-Smirnov test. Quantitative data were described as mean \pm standard deviation and median and range. Significance of the results was judged at the 5\% level. Student's $t$-test was used for normally distributed quantitative variables. The $\chi^{2}$ test was used for categorical variables. Fisher's exact or Monte Carlo correction was used when more than $20 \%$ of the cells had an expected count less than 5 .

\section{Results}

All subjects were infected by genotype $4 \mathrm{HCV}$. Their age range was $42-80$ years (mean $=55.3 \pm 9.3$ years). $77 \%$ (40 subjects) were male. Regarding characteristics before ablation: platelet count range was 46-259 $\times 10^{3} / \mathrm{mm}^{3}\left(119 \pm 58.4 \times 10^{3}\right)$, total bilirubin ranged from 0.5 to $2.1(1.18 \pm 0.42) \mathrm{mg} / \mathrm{dl}$. Albumin range was $2-4.5(3.4 \pm 0.46) \mathrm{g} / \mathrm{dl}$. AFP ranged from 0.6 to $1370 \mathrm{ng} / \mathrm{ml}$ (median = 75.5). Number of lesions ranged from 1 to $3(1.23 \pm 0.51)$ and size of largest lesion ranged from 1.7 to $5 \mathrm{~cm}(3.24 \pm 0.85) .11 .5 \%$ had mild ascites while others had no ascites. Performance status was 0 in $69.2 \%$ of patients and 1 in the remaining 30.8\%. MELD score range was 6-11 $(8.7 \pm 1.85)$. Child-Pugh score range was 5-8 $(5.88 \pm 0.94)$.

Thirty-eight patients received sofosbuvir plus daclatasvir ( \pm ribavirin) while the other 14 patients received sofosbuvir/ledipasvir ( \pm ribavirin). The period between the HCC ablation procedure and DAA initiation ranged from 4 to 32 weeks ( mean $=12.75$ weeks). Characteristics of included subjects in relation to time of DAA initiation are shown in Table 1.
Twenty-six patients (68\%) among those who received sofosbuvir + daclatasvir ( \pm ribavirin) reached a sustained virologic response at week 12 (SVR12) while 8 patients experienced viral relapse (21\%). Two patients died and the other two were lost to follow-up before assessment of SVR12 status. On the other hand, twelve patients $(85.7 \%)$ among those who received sofosbuvir/ledipasvir ( \pm ribavirin) showed SVR12 while no one experienced viral relapse. Two subjects were lost to follow-up before assessment for SVR12.

During the first year after ablation, 26.9\% (14 out of 52 patients) experienced tumor recurrence and by the end of the second year $42.3 \%$ ( 22 out of 52 patients) experienced tumor recurrence while 32 patients $(61.5 \%)$ did not experience any tumor recurrence until the end of the first year and 24 patients (46\%) did not experience this until the end of the second year. Two subjects died and 4 subjects were lost to follow-up during the first year before any tumor recurrence.

Thirty-six patients started DAAs within 6 months from HCC ablation, 8 of them experienced tumor recurrence within this period $(22.2 \%)$ while none of the 16 patients who started DAAs after 24 weeks from tumor ablation experienced any tumor recurrence during the first 6 months from ablation $(p=0.06)$ (Table 2). All of these 8 subjects had started DAAs just 4 weeks after tumor ablation. The recurrence was multicentric in $50 \%$ of them.

On comparing tumor recurrence between those who initiated HCV treatment earlier than 12 weeks from the tumor ablation procedure and those who initiated HCV treatment after 12 weeks from HCC ablation, the 6-month recurrence rate was $25 \%$ in the first group (8 out of 32 ), while no one in the second group experienced recurrence during the first 6 months after ablation $(p=0.013)$. But this difference was not maintained thereafter; tumor recurrence during the first year after ablation occurred in $31.3 \%$ of the first group (10 out of 32 ) and $20 \%$ in the second group ( 4 out of 20 ) $(p=0.591)$ while by the end of 2 years, $43.8 \%$ of the first group (14 out of 32) experienced tumor recurrence compared to $40 \%$ of the second group ( 8 out of 20 ) $(p=0.722)$ (Table 2). The higher recurrence rate during the first 6 months from ablation in the first group may be related to the significantly higher number of HCC lesions from the start $(p=0.002)$ (Table 1$)$.

\section{Discussion}

Hepatocellular carcinoma recurrence rates after surgical management and thermal ablation range widely among different studies. A meta-analysis assessing the HCC recurrence in $\mathrm{HCV}$ untreated pa- 
Table 1. Characteristics of included subjects in relation to time of directly acting antiviral (DAA) initiation

\begin{tabular}{|c|c|c|c|c|}
\hline \multirow[t]{2}{*}{ Parameter } & \multicolumn{4}{|c|}{ Time of DAA initiation from tumor ablation } \\
\hline & $\begin{array}{c}<12 \text { weeks } \\
(n=32)\end{array}$ & $\begin{array}{c}\geqslant 12 \text { weeks } \\
(n=20)\end{array}$ & $\begin{array}{c}<24 \text { weeks } \\
(n=36)\end{array}$ & $\begin{array}{c}\geqslant 24 \text { weeks } \\
\quad(n=16)\end{array}$ \\
\hline \multicolumn{5}{|l|}{ Age } \\
\hline Mean \pm SD & $57.1 \pm 10.6$ & $52.6 \pm 6.0$ & $56.5 \pm 10.5$ & $52.8 \pm 5.2$ \\
\hline$t(p)$ & \multicolumn{2}{|c|}{$1.938(0.058)$} & \multicolumn{2}{|c|}{$1.720(0.092)$} \\
\hline \multicolumn{5}{|l|}{ Platelet count $\left(/ \mathrm{mm}^{3}\right)$} \\
\hline Mean \pm SD & $128.7 \pm 59.0$ & $104.2 \pm 55.7$ & $123.9 \pm 57.7$ & $108.9 \pm 60.5$ \\
\hline$t(p)$ & \multicolumn{2}{|c|}{$1.488(0.143)$} & \multicolumn{2}{|c|}{$0.853(0.398)$} \\
\hline \multicolumn{5}{|l|}{ Total bilirubin (mg/dl) } \\
\hline Mean \pm SD & $1.2 \pm 0.5$ & $1.2 \pm 0.4$ & $1.2 \pm 0.4$ & $1.1 \pm 0.4$ \\
\hline$t(p)$ & \multicolumn{2}{|c|}{$0.335(0.739)$} & \multicolumn{2}{|c|}{$1.025(0.310)$} \\
\hline \multicolumn{5}{|l|}{ Serum albumin (g/dl) } \\
\hline Mean $\pm S D$ & $3.4 \pm 0.6$ & $3.4 \pm 0.3$ & $3.4 \pm 0.5$ & $3.5 \pm 0.3$ \\
\hline$t(p)$ & \multicolumn{2}{|c|}{$0(0.1000)$} & \multicolumn{2}{|c|}{$0.581(0.564)$} \\
\hline \multicolumn{5}{|l|}{ AFP before HCC ablation } \\
\hline Mean \pm SD & $281.1 \pm 435.9$ & $199.9 \pm 369.3$ & $332.1 \pm 467.5$ & $65 \pm 90.6$ \\
\hline$t(p)$ & \multicolumn{2}{|c|}{$0.692(0.492)$} & \multicolumn{2}{|c|}{$3.292^{*}\left(0.002^{*}\right)$} \\
\hline \multicolumn{5}{|l|}{ Total HBcAb } \\
\hline Negative & $27(84.4 \%)$ & $18(90 \%)$ & $31(86.1 \%)$ & $14(87.5 \%)$ \\
\hline Positive & $5(15.6 \%)$ & $2(10 \%)$ & $5(13.9 \%)$ & $2(12.5 \%)$ \\
\hline$\chi^{2}\left({ }^{\mathrm{FE}} p\right)$ & \multicolumn{2}{|c|}{$0.334(0.694)$} & \multicolumn{2}{|c|}{$0.018(1.000)$} \\
\hline \multicolumn{5}{|c|}{ Number of treated HCC lesions } \\
\hline Mean \pm SD & $1.4 \pm 0.6$ & $1 \pm 0$ & $1.3 \pm 0.6$ & $1 \pm 0$ \\
\hline$t(p)$ & \multicolumn{2}{|c|}{$3.483\left(0.002^{*}\right)$} & \multicolumn{2}{|c|}{$3.416^{*}\left(0.002^{*}\right)$} \\
\hline Size of largest lesion befor & & & & \\
\hline Mean \pm SD & $3.4 \pm 0.9$ & $3.0 \pm 0.7$ & $3.4 \pm 0.9$ & $3.0 \pm 0.8$ \\
\hline$t(p)$ & & & & \\
\hline Performance status $^{\dagger}$ & & & & \\
\hline 0 & $20(62.5 \%)$ & $16(80 \%)$ & $24(66.7 \%)$ & $12(75 \%)$ \\
\hline 1 & $12(37.5 \%)$ & $4(20 \%)$ & $12(33.3 \%)$ & $4(25 \%)$ \\
\hline$\chi^{2}(p)$ & & & 0.3 & \\
\hline MELD score & & & & \\
\hline Mean \pm SD & $8.8 \pm 2.0$ & $8.6 \pm 1.7$ & $8.8 \pm 1.9$ & $8.5 \pm 1.9$ \\
\hline$t(p)$ & & & & \\
\hline Child-Pugh score & & & & \\
\hline Median (min.-max.) & $6(5-8)$ & $6(5-7)$ & $6(5-8)$ & $5.5(5-7)$ \\
\hline Mean \pm SD & $5.9 \pm 1.0$ & $5.8 \pm 0.8$ & $5.9 \pm 1.0$ & $5.8 \pm 0.9$ \\
\hline$t(p)$ & & & & \\
\hline Type of antiviral therapy & & & & \\
\hline Sofosbuvir/ledipasvir & $8(25 \%)$ & $6(30 \%)$ & $10(27.8 \%)$ & $4(25 \%)$ \\
\hline Sofosbuvir/daclatasvir & $24(75 \%)$ & $14(70 \%)$ & $26(72.2 \%)$ & $12(75 \%)$ \\
\hline$\chi^{2}(p)$ & & & 0.0 & \\
\hline SVR12 & & & & \\
\hline Yes & $24(75 \%)$ & $14(70 \%)$ & $28(77.8 \%)$ & $10(62.5 \%)$ \\
\hline Died & $2(6.3 \%)$ & $0(0 \%)$ & $2(5.6 \%)$ & $0(0 \%)$ \\
\hline Relapse (no SVR) & $4(12.5 \%)$ & $4(20 \%)$ & $4(11.1 \%)$ & $4(25 \%)$ \\
\hline Lost & $2(6.3 \%)$ & $2(10 \%)$ & $2(5.6 \%)$ & $2(12.5 \%)$ \\
\hline$\left.\chi^{2}{ }^{\left({ }^{M C}\right.} p\right)$ & & & & \\
\hline
\end{tabular}

DAAs - directly acting antivirals, AFP - $\alpha$-fetoprotein, HBCAb - hepatitis B core antibody, MELD - Model of End Stage Liver Disease, SVR12 - sustained virological response 12 weeks after end of DAA treatment

$\chi^{2}$ - chi-square test, MC - Monte Carlo, FE - Fisher exact, $t$ - Student's t-test, * statistically significant at $p \leqslant 0.05, t$ - according to Eastern Cooperative Oncology Group (ECOG) scale 
Table 2. Relation between time of directly acting antiviral (DAA) initiation and hepatocellular carcinoma (HCC) recurrence

\begin{tabular}{|c|c|c|c|c|}
\hline \multirow[t]{2}{*}{$\mathrm{HCC}$ recurrence } & \multicolumn{4}{|c|}{ Time of DAA initiation from tumor ablation } \\
\hline & $<12$ weeks & $\geqslant 12$ weeks & $<24$ weeks & $\geqslant 24$ weeks \\
\hline 6 months & \multicolumn{2}{|c|}{$(n=52)$} & \multicolumn{2}{|c|}{$(n=52)$} \\
\hline No & $22(68.8 \%)$ & $20(100 \%)$ & $26(72.2 \%)$ & $16(100 \%)$ \\
\hline Yes & $8(25.0 \%)$ & $0(0 \%)$ & $8(22.2 \%)$ & $0(0 \%)$ \\
\hline Lost to follow-up & $2(6.3 \%)$ & $0(0 \%)$ & $2(5.6 \%)$ & $0(0 \%)$ \\
\hline$\chi^{2}\left({ }^{M C} p\right)$ & \multicolumn{2}{|c|}{$7.652^{*}\left(0.013^{*}\right)$} & \multicolumn{2}{|c|}{$5.120(0.060)$} \\
\hline 1 year & \multicolumn{2}{|c|}{$(n=52)$} & \multicolumn{2}{|c|}{$(n=52)$} \\
\hline No & $18(56.3 \%)$ & $14(70.0 \%)$ & $22(61.1 \%)$ & $10(62.5 \%)$ \\
\hline Yes & $10(31.3 \%)$ & $4(20.0 \%)$ & $10(27.8 \%)$ & $4(25.0 \%)$ \\
\hline Died & $2(6.3 \%)$ & $0(0 \%)$ & $2(5.6 \%)$ & $0(0 \%)$ \\
\hline Lost to follow-up & $2(6.3 \%)$ & $2(10.0 \%)$ & $2(5.6 \%)$ & $2(12.5 \%)$ \\
\hline$\chi^{2}\left({ }^{M C} p\right)$ & \multicolumn{2}{|c|}{$2.167(0.591)$} & \multicolumn{2}{|c|}{$1.465(0.808)$} \\
\hline 2 years & \multicolumn{2}{|c|}{$(n=52)$} & \multicolumn{2}{|c|}{$(n=52)$} \\
\hline No & $14(43.8 \%)$ & $10(50.0 \%)$ & $18(50.0 \%)$ & $6(37.5 \%)$ \\
\hline Yes & $14(43.8 \%)$ & $8(40.0 \%)$ & $14(38.9 \%)$ & $8(50.0 \%)$ \\
\hline Died & $2(6.3 \%)$ & $0(0.0 \%)$ & $2(5.6 \%)$ & $0(0.0 \%)$ \\
\hline Lost to follow-up & $2(6.3 \%)$ & $2(10.0 \%)$ & $2(5.6 \%)$ & $2(12.5 \%)$ \\
\hline$\chi^{2}(p)$ & \multicolumn{2}{|c|}{$1.343(0.722)$} & \multicolumn{2}{|c|}{$2.112(0.582)$} \\
\hline
\end{tabular}

DAAs - directly acting antivirals, HCC - hepatocellular carcinoma, $\chi^{2}$ - chi-square test, MC - Monte Carlo, *statistically significant at $p \leqslant 0.05$

tients after surgical resection or RFA for HCC lesions $<3 \mathrm{~cm}$ was conducted [18]. Among the included 11 studies, the 6-month recurrence rates ranged from $0 \%$ to $12.5 \%$, recurrence at 1 year ranged from $4.9 \%$ to $62.5 \%$ while recurrence at 2 years ranged from $31.8 \%$ to $100 \%$. Probabilities of recurrence were $7.4 \%$ at 6 months, $20 \%$ at 1 year and $47 \%$ at 2 years.

It is well known that antiviral therapy against $\mathrm{HBV}$ has a well-documented role in decreasing the HCC recurrence [3]. Unfortunately, the condition is different regarding $\mathrm{HCV}$. Many trials have studied the impact of HCV treatment using pegylated interferon on HCC recurrence [4]. The long-term studies have found a reduction in the incidence of HCC over time as a result of switch-off of the necro-inflammatory process and inflammatory cytokines, but without complete elimination of the risk in cirrhotic patients, and so longterm follow-up is necessary [5].

Much higher SVR rates are currently achieved thanks to DAAs. However, the impact of DAAs on HCC recurrence is debatable. The HCC recurrence rates after DAAs ranged among different studies from 0 to $54.4 \%$ [19].

Reig et al. [7] were the first to report high recurrence rates of HCC after receiving DAAs for $\mathrm{HCV}$. They reported recurrence of HCC in $28 \%$ of patients who received DAAs with median time for this being
3.5 months after initiating treatment with DAAs. Six patients had recurrence within 2 weeks from starting antiviral treatment. They also reported more aggressive HCC behavior [20]. Conti et al. [8] also reported HCC recurrence in $28 \%$ of cases within 6 months from completing DAAs therapy. Another study reported HCC recurrence in $30 \%$ of cases within 1 year from initiating DAA therapy [21]. Another European study [22] showed a high HCC recurrence rate after DAAs. Yang et al. [23] also reported higher HCC recurrence after liver transplantation in those who received DAAs while they were waiting for their surgery. In addition, El Kassas et al. [24], who studied HCC recurrence after DAA exposure among Egyptian patients with predominant genotype $4 \mathrm{HCV}$, reported a significantly increased HCC recurrence rate after DAA exposure in comparison to those who did not receive DAAs, but this study was a non-randomized one. Bielen et al. [25] reported high rates of HCC recurrence after DAAs (15\%) but they reported lower SVR in the group of HCC recurrence. In contrast to Yang et al. they reported no HCC recurrence in the group managed by liver transplantation, unlike those treated with RFA or liver resection. Renzulli et al. [26] reported that HCC nodules that occurred or recurred after DAAs showed higher microvascular invasion against tumors that preceded DAAs. 
Carcinogenesis after DAA therapy is suggested to be related to HCV-induced dysregulation between cell apoptosis and prosurvival [27, 28]. Tumor emergence after DAAs may be precipitated by the direct and rapid viral clearance irrespective of the immune system status, thereby disrupting the immune cancer surveillance and leading to progression of dormant tumor cells [28, 29]. Rapid viral eradication results in disturbance of innate immunity and decrease in type II and III receptors of interferon, which regulates tumor angiogenesis in addition to its anti-proliferative effect $[19,29]$. Also rapid reduction in natural killer cells has been suggested to be a possible cause of rapid HCC progression $[29,30]$. Twelve immune mediators showed higher levels before DAA initiation in patients who developed HCC thereafter [31]. Moreover, the level of tumor necrosis factor $\alpha$ (TNF- $\alpha$ ) remained high after DAA completion in those who developed HCC thereafter and decreased in subjects who did not develop HCC later, which suggests a role for persistent high TNF- $\alpha$ in HCC occurrence and recurrence after DAAs [31, 32]. Villani et al. [10] demonstrated that vascular endothelial growth factor (VEGF) elevated during DAA therapy and remained high for 3 months afterwards. Faillaci et al. [33] found higher levels of angiopoietin-2 among persons with recurrent and new HCC, and they demonstrated that the increase in VEGF caused by DAAs "acts as a trigger" in patients who have clinically significant portal hypertension, which leads to activation of neo-angiogenesis in the liver.

On the other hand, the ANRS study which involved 3 French cohorts of HCV patients with ablated HCC showed no differences regarding HCC recurrence in those who received DAAs and those who did not [13]. Another multicenter study showed no elevation in HCC recurrence on DAAs [34]. Cabibbo et al. [35] reported that the 6-month and 1-year HCC recurrence rates after DAAs were comparable to the risk reported previously before the era of DAAs ( $12 \%$ and $26.6 \%$, respectively). Beste et al. [36] also found no elevated risk of HCC recurrence on DAAs.

Huang et al. [37] reported that patients of the DAA group had a lower risk of dropout from the transplantation waiting list due to death or HCC growth compared to the untreated group.

Also, Singal et al. [38] reported that DAAs were not linked to HCC recurrence in their studied multicenter North American cohort. A Japanese study showed no variance in the pattern and risk of HCC recurrence between those who received interferon and those who received DAAs [39]. A meta-analysis found no proof of elevated risk of HCC recurrence after DAAs [40]. An- other study reported improved survival in successfully treated early HCC patients who received DAAs [41].

Zeng et al. [42] reported no tumor recurrence in the HCC group. Another study reported that DAA treatment significantly decreased HCC recurrence rates compared with the untreated patients $[43,44]$. A more recent study also reported the same conclusion [45].

In a study that included 204 patients from 16 liver centers in Poland with 2-year post-DAA follow-up, 3 out of 10 patients with documented history of HCC before starting DAAs experienced HCC recurrence in addition to another 4 patients who developed de novo HCC occurrence [46].

In the current study, $26.9 \%$ of patients experienced tumor recurrence in the first year, which increased to $42.3 \%$ by the end of the second year. This is very similar to the findings of a meta-analysis performed by Cabibbo et al., who found probabilities of HCC recurrence in HCV untreated patients to be $20 \%$ at 1 year and $47 \%$ at 2 years, although the subjects included in that meta-analysis were only Child-Pugh A patients with lesions less than $3 \mathrm{~cm}$ and treated using either surgical resection or RFA [18].

In our patients, the SVR12 rate among those who received sofosbuvir + daclatasvir was $68 \%$ while $85.7 \%$ of those who received sofosbuvir/ledipasvir reached SVR12. Different studies demonstrate a lower SVR12 after DAAs in those with underlying HCC. Beste et al. [36] reported SVR12 in $74 \%$ of HCC patients compared with $91 \%$ in those without HCC. Prenner et al. [47] reported higher viral relapse in HCC patients compared to nonHCC patients (21\% vs. $12 \%)$. Ji et al. [48] reported lower SVR12 among HCC patients infected by genotype 1 $\mathrm{HCV}$ treated with ledipasvir/sofosbuvir compared with non-HCC patients. The SVR12 rate in the HCC group was $94.1 \%$ (95\% CI: $89.9-97.3)$ vs. $98.7 \%$ (95\% CI: 97.5-99.6) in non-HCC patients. Hassany et al. [49] compared SVR rates in those with successfully treated HCC after using sofosbuvir with ribavirin or sofosbuvir in addition to either simeprevir or daclatasvir. They reported the overall SVR 12 rate to be $64.5 \%$, but it was highest in the sofosbuvir plus daclatasvir with ribavirin group (87.5\%), which is noticeably higher than SVR12 rates achieved using the same drugs in our cohort. This might point to the importance of adding ribavirin to all patients with ablated HCC using sofosbuvir plus daclatasvir for HCV treatment even in those within ChildPugh class A.

Lower SVR rates among HCC patients are suspected to be due to impaired delivery of the drug to the HCC area and so HCC foci can act as a reservoir for viral replication. Also immune and inflammatory al- 
terations in the HCC background can have a role in this higher HCV relapse after DAAs [47, 50].

A Japanese study recommended at least a 120-day interval between HCC curative management and starting DAAs to decrease HCC recurrence [51]. Higher 6-month HCC recurrence was observed in our study in those who started DAAs within 12 weeks from tumor management, but this may be related to a significantly higher number of malignant foci from the start in that group. Also this higher recurrence was not maintained at 1 and 2 years.

Limitations of the current study are the relatively small sample size and being a retrospective study. Strengths of our study include the long (2-year) follow-up period, and starting the follow-up from the time of tumor ablation, unlike other studies which started the follow-up from the time of DAA initiation or completion. The period between the HCC ablation procedure and the DAA initiation ranged from 4 to 32 weeks ( mean $=12.75$ weeks). This makes comparison with data reported before the era of DAAs applicable.

\section{Conclusions}

In conclusion, although our study included both modified Child-Pugh A and B patients and included lesions up to $5 \mathrm{~cm}$ treated using thermal ablation, the 1-year and 2-year HCC recurrence rates were similar to those previously reported for surgical resection or radiofrequency ablation of lesions up to $3 \mathrm{~cm}$ in ChildPugh A patients before development of directly acting antivirals, and so we can conclude that DAA usage after confirming complete HCC ablation using the triphasic CT liver scan does not increase HCC recurrence.

\section{Acknowledgements}

The authors acknowledge Dr. Dalia K. El Deeb and Dr. Mona H. Ashry for their assistance in statistical analysis. Also, we acknowledge Aya M. Abdelaziz for her technical support.

\section{Disclosure}

The authors declare no conflict of interest.

\section{References}

1. Kudo M. Recent trends in the management of hepatocellular carcinoma with special emphasis on treatment with regorafenib and immune checkpoint inhibitors. Dig Dis 2016; 34: 714-730.

2. Ibrahim AS, Khaled HM, Mikhail NN, et al. Cancer incidence in Egypt: results of the national population-based cancer registry program. J Cancer Epidemiol 2014; 2014: 437971.
3. Yang S, Lin Q, Lin W, et al. Effect of adjuvant interferon therapy on hepatitis B virus-related hepatocellular carcinoma: a systematic review. World J Surg Oncol 2016; 14: 159.

4. Hsu CS, Chao YC, Lin HH, et al. Systematic review: impact of interferon-based therapy on HCV-related hepatocellular carcinoma. Sci Rep 2015; 5: 9954.

5. D’Ambrosio R, Della Corte C, Colombo M. Hepatocellular carcinoma in patients with a sustained response to anti-hepatitis $\mathrm{C}$ therapy. Intern J Mol Sci 2015; 16: 19698-19712.

6. Reig M, Boix L, Bruix J. The impact of direct antiviral agents on the development and recurrence of hepatocellular carcinoma. Liver Intern 2017; 37: 136-139.

7. Reig M, Marino Z, Perello C, et al. Unexpected high rate of early tumor recurrence in patients with HCV-related HCC undergoing interferon-free therapy. J Hepatol 2016; 65: 719-726.

8. Conti F, Buonfiglioli F, Scuteri A, et al. Early occurrence and recurrence of hepatocellular carcinoma in HCV-related cirrhosis treated with direct-acting antivirals. J Hepatol 2016; 65: 727-733.

9. Kozbial K, Moser S, Schwarzer R, et al. Unexpected high incidence of hepatocellular carcinoma in cirrhotic patients with sustained virologic response following interferon-free direct-acting antiviral treatment. J Hepatol 2016; 65: 856-858.

10. Villani R, Facciorusso A, Bellanti F, et al. DAAs rapidly reduce inflammation but increase serum VEGF level: a rationale for tumor risk during anti-HCV treatment. PLoS One 2016; 11: e0167934.

11. Minami T, Tateishi R, Nakagomi R, et al. The impact of direct-acting antivirals on early tumor recurrence after radiofrequency ablation in hepatitis C-related hepatocellular carcinoma. J Hepatol 2016; 65: 1272-1273.

12. Manthravadi S, Paleti S, Pandya P. Impact of sustained viral response postcurative therapy of hepatitis C-related hepatocellular carcinoma: a systematic review and meta-analysis. Intern J Cancer 2017; 140: 1042-1049.

13. ANRS collaborative study group on hepatocellular carcinoma. Lack of evidence of an effect of direct-acting antivirals on the recurrence of hepatocellular carcinoma: data from three ANRS cohorts. J Hepatol 2016; 65: 734-740.

14. Kamal A, Elmoety AAA, Rostom YAM, et al. Percutaneous radiofrequency versus microwave ablation for management of hepatocellular carcinoma: a randomized controlled trial. J Gastrointest Oncol 2019; 10: 562-571

15. Mitchell DG, Bruix J, Sherman M, et al. LI-RADS (Liver Imaging Reporting and Data System): summary, discussion, and consensus of the LI-RADS Management Working Group and future directions. Hepatology 2015; 61: 1056-1065.

16. Pawlotsky JM, Aghemo A, Back D, et al. EASL recommendations on treatment of hepatitis C 2015. J Hepatol 2015; 63: 199236.

17. Lencioni R, Llovet JM. Modified RECIST (mRECIST) assessment for hepatocellular carcinoma. Semin Liver Dis 2010; 30: 52-60.

18. Cabibbo G, Petta $S$, Barbàra $M$, et al. A meta-analysis of single $\mathrm{HCV}$-untreated arm of studies evaluating outcomes after curative treatments of HCV-related hepatocellular carcinoma. Liver Intern 2017; 37: 1157-1166.

19. Guarino M, Sessa A, Cossiga V, et al. Direct-acting antivirals and hepatocellular carcinoma in chronic hepatitis C: A few lights and many shadows. World J Gastroenterol 2018; 24: 2582-2595.

20. Reig M, Mariño Z, Perelló C, et al. Tumour recurrence after Interferon-free treatment for hepatitis $\mathrm{C}$ in patients with previously treated hepatocellular carcinoma discloses a more aggressive 
pattern and faster tumour growth. Journal Hepatol 2017; 66: S20.

21. Calleja JL, Crespo J, Rincon D, et al. Effectiveness, safety and clinical outcomes of direct-acting antiviral therapy in $\mathrm{HCV}$ genotype 1 infection: Results from a Spanish real-world cohort. J Hepatol 2017; 66: 1138-1148.

22. Kolly P, Waidmann O, Vermehren J, et al. Hepatocellular carcinoma recurrence after direct antiviral agent treatment: A European multicentre study. J Hepatol 2017; 67: 876-878.

23. Yang JD, Aqel BA, Pungpapong S, et al. Direct acting antiviral therapy and tumor recurrence after liver transplantation for hepatitis C-associated hepatocellular carcinoma. J Hepatol 2016; 65: 859-860.

24. El Kassas M, Funk A, Salaheldin M, et al. Increased recurrence rates of hepatocellular carcinoma after DAA therapy in a hepatitis C-infected Egyptian cohort: A comparative analysis. J Viral Hepat 2018; 25: 623-630.

25. Bielen R, Moreno C, Van Vlierberghe H, et al. The risk of early occurrence and recurrence of hepatocellular carcinoma in hepatitis $\mathrm{C}$-infected patients treated with direct-acting antivirals with and without pegylated interferon: A Belgian experience. J Viral Hepat 2017; 24: 976-981.

26. Renzulli M, Buonfiglioli F, Conti F, et al. Imaging features of microvascular invasion in hepatocellular carcinoma developed after direct-acting antiviral therapy in HCV-related cirrhosis. Eur Radiol 2018; 28: 506-513.

27. Hayes CN, Zhang P, Zhang Y, et al. Molecular mechanisms of hepatocarcinogenesis following sustained virological response in patients with chronic hepatitis $\mathrm{C}$ virus infection. Viruses 2018; 10: E531.

28. Sghaier I, Brochot E, Loueslati BY, et al. Hepatitis C virus protein interaction network for HCV clearance and association of DAA to HCC occurrence via data mining approach: A systematic review and critical analysis. Rev Med Virol 2019; 6: E2033.

29. Villani R, Vendemiale G, Serviddio G. Molecular mechanisms involved in $\mathrm{HCC}$ recurrence after direct-acting antiviral therapy. Int J Mol Sci 2018; 20: E49.

30. Chu PS, Nakamoto N, Taniki N, et al. On-treatment decrease of NKG2D correlates to early emergence of clinically evident hepatocellular carcinoma after interferon-free therapy for chronic hepatitis C. PLoS One 2017; 12: E0179096.

31. Debes JD, van Tilborg M, Groothuismink ZMA, et al. Levels of cytokines in serum associate with development of hepatocellular carcinoma in patients with HCV infection treated with direct-acting antivirals. Gastroenterology 2018; 154: 515-517.

32. Roche B, Coilly A, Duclos-Vallee JC, Samuel D. The impact of treatment of hepatitis $\mathrm{C}$ with DAAs on the occurrence of HCC. Liver Int 2018; 1: 139-145.

33. Faillaci F, Marzi L, Critelli R, et al. Liver angiopoietin-2 is a key predictor of de novo or recurrent hepatocellular cancer after hepatitis C virus direct-acting antivirals. Hepatology 2018; 68: 1010-1024

34. Ogawa E, Furusyo N, Nomura H, et al. Short-term risk of hepatocellular carcinoma after hepatitis $\mathrm{C}$ virus eradication following direct-acting anti-viral treatment. Aliment Pharmacol Ther 2018; 47: 104-113.

35. Cabibbo G, Petta $S$, Calvaruso V, et al. Is early recurrence of hepatocellular carcinoma in HCV cirrhotic patients affected by treatment with direct-acting antivirals? A prospective multicentre study. Aliment Pharmacol Ther 2017; 46: 688-695.

36. Beste LA, Green PK, Berry K, et al. Effectiveness of hepatitis C antiviral treatment in a USA cohort of veteran patients with hepatocellular carcinoma. J Hepatol 2017; 67: 32-39.
37. Huang AC, Mehta N, Dodge JL, et al. Direct-acting antivirals do not increase the risk of hepatocellular carcinoma recurrence after local-regional therapy or liver transplant waitlist dropout. Hepatology 2018; 68: 449-461.

38. Singal AG, Rich NE, Mehta N, et al. Direct-acting antiviral therapy not associated with recurrence of hepatocellular carcinoma in a multicenter North American Cohort Study. Gastroenterology 2019; 156: 1683-92.e1.

39. Nishibatake Kinoshita M, Minami T, Tateishi R, et al. Impact of direct-acting antivirals on early recurrence of HCV-related HCC: Comparison with interferon-based therapy. J Hepatol 2019; 70: 78-86.

40. Rutledge SM, Zheng H, Li DK, et al. No evidence for higher rates of hepatocellular carcinoma after direct-acting antiviral treatment: a meta-analysis. Hepatoma Res 2019; 5: 31.

41. Cabibbo G, Celsa C, Calvaruso V, et al. Direct-acting antivirals after successful treatment of early hepatocellular carcinoma improve survival in HCV-cirrhotic patients. J Hepatol 2019; 71: 265-273.

42. Zeng QL, Li ZQ, Liang HX, et al. Unexpected high incidence of hepatocellular carcinoma in patients with hepatitis $C$ in the era of DAAs: Too alarming? J Hepatol 2016; 65: 1068-1069.

43. Ikeda K, Kawamura Y, Kobayashi M, et al. Direct-acting antivirals decreased tumor recurrence after initial treatment of hepatitis C virus-related hepatocellular carcinoma. Dig Dis Sci 2017; 62: 2932-2942.

44. Virlogeux V, Pradat P, Hartig-Lavie K, et al. Direct-acting antiviral therapy decreases hepatocellular carcinoma recurrence rate in cirrhotic patients with chronic hepatitis C. Liver Int 2017; 37: $1122-1127$

45. Imai K, Takai K, Hanai T, et al. Sustained virological response by direct-acting antivirals reduces the recurrence risk of hepatitis C-related hepatocellular carcinoma after curative treatment. Mol Clin Oncol 2020; 12: 111-116.

46. Flisiak R, Janczewska E, Łucejko M, et al. Durability of virologic response, risk of de novo hepatocellular carcinoma, liver function and stiffness 2 years after treatment with ombitasvir/ paritaprevir/ritonavir \pm dasabuvir \pm ribavirin in the AMBER, real-world experience study. J Viral Hepat 2018; 25: 1298-1305.

47. Prenner SB, VanWagner LB, Flamm SL, et al. Hepatocellular carcinoma decreases the chance of successful hepatitis $C$ virus therapy with direct-acting antivirals. J Hepatol 2017; 66: 1173-1181.

48. Ji F, Wei MT, Yeo YH, et al. Sustained virologic response (SVR) to direct-acting antiviral (DAA) therapy in patients with chronic hepatitis $\mathrm{C}$ virus (HCV) infection and hepatocellular carcinoma (HCC): a systematic review and meta-analysis. J Hepatol 2018; 68: S259-260.

49. Hassany M, Elsharkawy A, Maged A, et al. Hepatitis C virus treatment by direct-acting antivirals in successfully treated hepatocellular carcinoma and possible mutual impact. Eur J Gastroenterol Hepatol 2018; 30: 876-881.

50. Syed T, Fazili JD, Ali IA, et al. Hepatocellular carcinoma occurrence and recurrence in hepatitis c-infected patients treated with direct-acting antivirals. Cureus 2018; 10: e2843.

51. Iida H, Osaki R, Fujimoto T, et al. Interval between hepatocellular carcinoma treatment and interferon-free direct-acting antiviral agents against hepatitis $\mathrm{C}$ is necessary to suppress tumor recurrence. Mol Clin Oncol 2019; 11: 99-105. 